\title{
Threshold Effects of Serum Uric Acid on Chronic Kidney Disease in US Women without Hypertension and Diabetes: A Cross-Sectional Study
}

\author{
Guiping $\mathrm{Hu}^{\mathrm{a}, \mathrm{b}}$ Yi Baic Tian Chen ${ }^{\mathrm{c}}$ Shichuan Tang ${ }^{\mathrm{d}}$ Lihua Hu $\mathrm{H}^{\mathrm{e}}$ \\ aSchool of Medicine, Beihang University, Beijing, China; beijing Advanced Innovation Center for \\ Big Data-Based Precision Medicine, Beihang University, Beijing, China; 'School of Public Health, \\ Capital Medical University, Beijing, China; ${ }^{d}$ Key Laboratory of Occupational Safety and Health, \\ Beijing Municipal Institute of Labor Protection, Beijing, China; ${ }^{\mathrm{e} D e p a r t m e n t ~ o f ~ C a r d i o v a s c u l a r ~}$ \\ Medicine, The Second Affiliated Hospital of Nanchang University, Nanchang, China
}

\section{Keywords}

Serum uric acid $\cdot$ Chronic kidney disease $\cdot$ Threshold effects · Women

\begin{abstract}
Background: Serum uric acid (SUA) has been associated with increased risk of chronic kidney disease (CKD) in observational studies; however, data in women without hypertension and diabetes are sparse. Purpose: To examine the association between SUA and CKD among women without hypertension and diabetes. Methods: In this cross-sectional study of 6,776 US women without hypertension and diabetes from the National Health and Nutrition Examination Survey (1999-2006), we investigated the relationship between SUA and CKD using multivariable logistic regression models. Moreover, a generalized additive model and smooth curve fitting (penalized spline method) and a 2 piecewise logistic regression models were conducted to address for nonlinearity. Results: The prevalence of CKD was $8.3 \%$. Multiple logistic analyses showed that per $1 \mathrm{mg} / \mathrm{dL}$ increase in SUA was associated with $39 \%$ increased prevalence of CKD. Analyses using restricted cubic spline confirmed that the association between SUA and CKD was nonlinear. Further, threshold and saturation effect analysis showed that the inflection point of SUA was $4.5 \mathrm{mg} / \mathrm{dL}$. The ORs (95\% Cls) were $0.84(0.66-1.08)$ on the left side of inflection point and $1.87(1.56-2.24)$ on the right side of inflection point, respectively. Subgroup analysis showed that the stronger association between SUA and CKD was observed in elder women with never/former smoking and higher fasting blood glucose levels (all $p$ values for interaction $<0.05$ ). Conclusion: Our study suggested threshold effects of SUA on the prevalence of CKD among US women without hypertension and diabetes. SUA levels $>4.5 \mathrm{mg} / \mathrm{dL}$ were positively and independently associated with CKD.
\end{abstract}




\section{Kidney \\ Blood Pressure \\ Research}

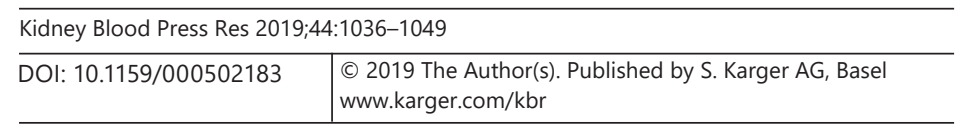

Hu et al.: Threshold Effects of SUA on CKD in Healthy Women

\section{Introduction}

Chronic kidney disease (CKD) is increasingly recognized as a global health issue [1]. The burden of CKD was not only restricted to progress to end-stage renal disease requiring renal replacement therapy but also associated with high mortality, cardiovascular events [2,3]. Several common risk factors that contribute to CKD development are hypertension, diabetes, obesity, and proteinuria [4,5]. Thus, in addition to controlling these risk factors, new and effective strategies are needed for preventing CKD development.

Serum uric acid (SUA), an end-product of purine metabolism, is associated with a wide variety of adverse health outcomes, including hypertension, atherosclerosis, cardiovascular disease, and mortality [6-8]. Multiple epidemiological studies have investigated the effects of SUA levels on CKD, with discrepant results [9-12]. Although some observational studies have confirmed that elevated SUA levels predict the development of CKD $[9,10]$, several studies failed to show a significant contribution of SUA to CKD development $[11,12]$. These conflicting results might be attributed to the differences in cohort characteristics, population, sample size, and adjustment of confounders. Furthermore, the development of CKD may be associated with hypertension and diabetes; thus, the association between SUA and CKD may not be reliable. However, to our knowledge, no study has discussed the association between SUA level and CKD in population without hypertension and diabetes. Additionally, previous studies only investigated the linear relationship between SUA and CKD, but not discuss the nonlinear relationship.

Therefore, given the possibility of developing CKD bias among population with diabetes and hypertension, we analyzed data from National Health and Nutrition Examination Survey (NHANES) and aimed to assess the association between SUA and CKD among US women without hypertension and diabetes.

\section{Methods}

\section{Study Design and Population}

NHANES, an ongoing repeated cross-sectional study conducted by the Centers for Disease Control and Prevention, is a nationwide database that contains information about the lifestyle, health, and nutrition status of adults and children in the United States. Participants answered questionnaires on demographics, lifestyle, and medical factors. NHANES also obtained anthropometric measurements and biomarker data at mobile examination centers. Written informed consent was obtained from all NHANES participants. The National Center for Health Statistics Research Ethics Review Board approved NHANES protocols and posting of the files for public use. More detailed information is available on the official website (http:// www.cdc.gov/nchs/nhanes/nhanes_questionnairees.htm). We download data for the analyses presented here from DataDryad (https://doi.org/10.5061/dryad.d5h62).

We performed a secondary analysis based on data of four 2-year NHANES survey cycles: 1999-2000, 2001-2002, 2003-2004, and 2005-2006. In total, 11,858 eligible women aged $\geq 18$ years were enrolled. Next, we screened participants according to the exclusion criteria listed below: (1) subjects with hypertension and diabetes $(n=4,137)$; (2) participants with missing CKD and SUA ( $n=940)$; and ( 3 ) use antihyperuricemic drugs $(n=5)$. Given the possibility of developing CKD bias among population with diabetes and hypertension, we excluded these patients in order to better observe the association between SUA and CKD. Finally, a total of 6,776 participants were analyzed (Fig. 1). 
Fig. 1. Flow chart of study participants. NHANES, National Health and Nutrition Examination Survey; CKD, chronic kidney disease; SUA, serum uric acid.

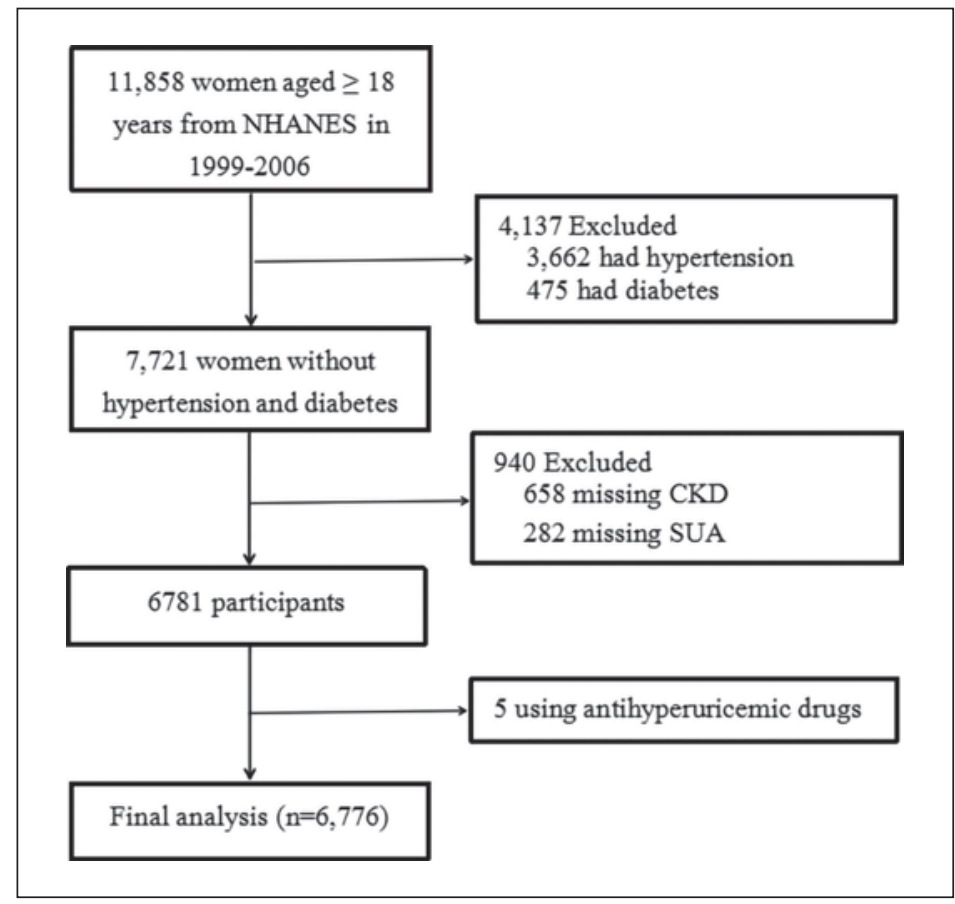

\section{Serum Uric Acid}

SUA was measured on a Roche Hitachi Model 917 or 704 Multichannel Analyzer in 19992001 and a Beckman Synchron LX20 in 2002-2006 using a colorimetric method. Biochemistry measurements were performed by the Coulston Foundation in New Mexico in 19992001 and by Collaborative Laboratory Services in Iowa in 2002-2006. The distribution of creatinine and uric acid results from the 2 laboratories were compared at the time of transition, and no significant differences were observed. The details of SUA measurement process and other covariate acquisition process are available at http://cdc.gov/nchs/nhanes.

\section{Definition of CKD}

Serum creatinine (Scr) was measured by the Jaffe reaction and standardized by methods described previously [13]. Estimated glomerular filtration rate (eGFR, $\mathrm{mL} / \mathrm{min}$ per $1.73 \mathrm{~m}^{2}$ ) was calculated via the newly developed CKD-EPI equation [14]: eGFR $=141 \times \min (\mathrm{Scr} / \kappa, 1)^{\alpha}$ $\times \max (\mathrm{Scr} / \kappa, 1)^{-1.209} \times 0.993^{\mathrm{Age}} \times 1.018$ (if female) $\times 1.159$ (if black), $\kappa$ is 0.7 for females and 0.9 for males, $\alpha$ is -0.329 for females and -0.411 for males, min indicates the minimum of $\mathrm{Scr} / \kappa$ or 1 , and max indicates the maximum of Scr/ $\kappa$ or 1 . A random urine specimen was collected from participants, and urinary creatinine was measured by the Jaffé rate reaction; urinary albumin was measured by solid-phase fluorescent immunoassay [15]. Albuminuria was measured by urinary albumin-creatinine ratio (ACR). We used the kidney disease classification system recommended by the KDIGO conference report [16], which accounts for both level of eGFR and albuminuria. As a simplified classification scheme, we defined CKD by either eGFR $<60 \mathrm{~mL} / \mathrm{min} / 1.73 \mathrm{~m}^{2}$ or albuminuria as urine ACR $\geq 30 \mathrm{mg} / \mathrm{g}$.

\section{Definition of Hypertension and Diabetes}

Hypertension was defined as a mean diastolic blood pressure (DBP) $\geq 90 \mathrm{~mm} \mathrm{Hg}$ or a mean systolic blood pressure (SBP) $\geq 140 \mathrm{~mm} \mathrm{Hg}$ or a positive response to the questions: "Have you been told on 2 or more different visits that you had hypertension?" or "Are you taking prescribed 
Table 1. Characteristics of study population

\begin{tabular}{|c|c|c|c|c|}
\hline \multirow[t]{2}{*}{ Characteristics ${ }^{*}$} & \multicolumn{3}{|l|}{ SUA, mg/dL } & \multirow[t]{2}{*}{$p$ value } \\
\hline & tertile $1(<4.5)$ & tertile $2(4.5-5.7)$ & tertile $3(\geq 5.7)$ & \\
\hline Number & 3,866 & 2,124 & 786 & \\
\hline Age, years & $38.4 \pm 14.3$ & $41.4 \pm 16.2$ & $45.0 \pm 18.4$ & $<0.001$ \\
\hline \multicolumn{4}{|l|}{ Race/ethnicity, \% } & 0.043 \\
\hline Non-Hispanic white & 70.0 & 72.0 & 71.6 & \\
\hline Non-Hispanic black & 10.0 & 9.8 & 11.4 & \\
\hline Mexican American & 8.7 & 7.3 & 5.0 & \\
\hline Other Hispanic & 6.4 & 5.8 & 6.7 & \\
\hline Other race & 5.0 & 5.1 & 5.3 & \\
\hline \multicolumn{4}{|l|}{ Education, \% } & $<0.001$ \\
\hline$<$ High school & 16.5 & 17.5 & 17.9 & \\
\hline High school & 22.8 & 25.5 & 29.1 & \\
\hline$>$ High school & 60.8 & 57.0 & 53.1 & \\
\hline \multicolumn{4}{|l|}{ Smoking, \% } & $<0.001$ \\
\hline Never & 60.1 & 54.8 & 52.1 & \\
\hline Former & 17.3 & 21.7 & 23.0 & \\
\hline Current & 22.6 & 23.5 & 24.9 & \\
\hline \multicolumn{4}{|l|}{ Physical activity, \%थ } & $<0.001$ \\
\hline Sedentary & 16.7 & 15.4 & 23.2 & \\
\hline Low & 28.4 & 28.4 & 33.0 & \\
\hline Moderate & 21.6 & 22.5 & 15.6 & \\
\hline High & 33.4 & 33.6 & 28.2 & \\
\hline Poverty to income ratio & $3.0 \pm 1.7$ & $2.9 \pm 1.7$ & $2.8 \pm 1.6$ & 0.002 \\
\hline \multicolumn{5}{|l|}{ Physical examination data } \\
\hline BMI, $\mathrm{kg} / \mathrm{m}^{2 \S}$ & $25.1 \pm 5.3$ & $28.3 \pm 6.3$ & $31.3 \pm 7.2$ & $<0.001$ \\
\hline SBP, mm Hg & $110.4 \pm 10.7$ & $113.1 \pm 11.3$ & $115.2 \pm 11.4$ & $<0.001$ \\
\hline DBP, mm Hg & $67.2 \pm 9.6$ & $69.1 \pm 9.1$ & $69.5 \pm 10.1$ & $<0.001$ \\
\hline \multicolumn{5}{|l|}{ Dietary } \\
\hline Alcohol, g & $5.6 \pm 17.0$ & $6.0 \pm 17.7$ & $8.9 \pm 25.3$ & $<0.001$ \\
\hline Caffeine, mg & $154.6 \pm 200.1$ & $172.9 \pm 188.2$ & $160.6 \pm 184.5$ & 0.003 \\
\hline Calcium, mg & $838.9 \pm 507.9$ & $771.8 \pm 456.1$ & $715.4 \pm 397.5$ & $<0.001$ \\
\hline Carbohydrate, g & $245.9 \pm 104.4$ & $234.1 \pm 103.7$ & $227.9 \pm 97.9$ & $<0.001$ \\
\hline Cholesterol, mg & $238.5 \pm 182.0$ & $241.2 \pm 180.5$ & $237.8 \pm 163.3$ & 0.833 \\
\hline Dietary fiber, g & $14.7 \pm 8.3$ & $13.7 \pm 7.4$ & $12.5 \pm 6.8$ & $<0.001$ \\
\hline Energy, kcal & $1,915.9 \pm 737.1$ & $1,862.2 \pm 719.7$ & $1,827.7 \pm 709.3$ & 0.001 \\
\hline TMFA, g & $26.4 \pm 13.7$ & $25.9 \pm 13.6$ & $24.7 \pm 12.2$ & 0.006 \\
\hline TPFA, g & $15.2 \pm 9.0$ & $15.1 \pm 9.2$ & $14.4 \pm 8.3$ & 0.085 \\
\hline TSFA, g & $23.7 \pm 12.8$ & $23.2 \pm 12.6$ & $22.3 \pm 11.4$ & 0.009 \\
\hline Total fat, $g$ & $71.4 \pm 34.9$ & $70.3 \pm 34.6$ & $67.2 \pm 31.5$ & 0.008 \\
\hline Protein, g & $70.2 \pm 30.4$ & $69.9 \pm 30.9$ & $68.6 \pm 28.9$ & 0.379 \\
\hline \multicolumn{5}{|l|}{ Laboratory data } \\
\hline Albumin, g/dL & $4.2 \pm 0.4$ & $4.2 \pm 0.3$ & $4.2 \pm 0.3$ & 0.005 \\
\hline ALT, U/L & $19.3 \pm 11.9$ & $20.8 \pm 11.8$ & $28.6 \pm 82.7$ & $<0.001$ \\
\hline AST, U/L & $21.4 \pm 8.1$ & $22.3 \pm 10.0$ & $25.4 \pm 14.6$ & $<0.001$ \\
\hline GGT, U/L & $18.1 \pm 21.8$ & $21.4 \pm 25.3$ & $30.5 \pm 39.2$ & $<0.001$ \\
\hline $\mathrm{ACR}, \mathrm{mg} / \mathrm{g}$ & $12.3 \pm 30.5$ & $13.2 \pm 64.8$ & $21.7 \pm 110.3$ & 0.001 \\
\hline eGFR, $\mathrm{mL} / \mathrm{min}$ per $1.73 \mathrm{~m}^{2}$ & $109.0 \pm 20.6$ & $100.7 \pm 22.0$ & $93.6 \pm 26.5$ & $<0.001$ \\
\hline SUA, mg/dL & $3.7 \pm 0.5$ & $5.0 \pm 0.3$ & $6.4 \pm 0.8$ & $<0.001$ \\
\hline $\mathrm{FBG}, \mathrm{mg} / \mathrm{dL}$ & $85.8 \pm 9.5$ & $88.0 \pm 10.7$ & $90.1 \pm 12.1$ & $<0.001$ \\
\hline 2-h glucose, $\mathrm{mg} / \mathrm{dL}$ & $94.7 \pm 27.1$ & $106.0 \pm 30.0$ & $119.3 \pm 35.2$ & $<0.001$ \\
\hline Glycated hemoglobin & $5.1 \pm 0.3$ & $5.2 \pm 0.4$ & $5.3 \pm 0.4$ & $<0.001$ \\
\hline Total cholesterol, mg/dL & $193.7 \pm 38.7$ & $203.3 \pm 43.2$ & $207.3 \pm 41.8$ & $<0.001$ \\
\hline Triglycerides, mg/dL & $101.6 \pm 63.7$ & $126.5 \pm 115.2$ & $155.0 \pm 105.5$ & $<0.001$ \\
\hline
\end{tabular}


Table 1 (continued)

\begin{tabular}{|c|c|c|c|c|}
\hline \multirow[t]{2}{*}{ Characteristics $^{*}$} & \multicolumn{3}{|l|}{$\mathrm{SUA}, \mathrm{mg} / \mathrm{dL}$} & \multirow[t]{2}{*}{$p$ value } \\
\hline & tertile $1(<4.5)$ & tertile $2(4.5-5.7)$ & tertile 3 ( $\geq 5.7$ ) & \\
\hline Homocysteine, $\mu \mathrm{mol} / \mathrm{L}$ & $6.8 \pm 2.3$ & $7.6 \pm 3.7$ & $8.8 \pm 5.6$ & $<0.001$ \\
\hline Serum folate, $\mathrm{ng} / \mathrm{mL}$ & $14.4 \pm 8.2$ & $14.4 \pm 8.3$ & $15.1 \pm 11.5$ & 0.120 \\
\hline CKD, \% & 5.3 & 7.3 & 18.1 & $<0.001$ \\
\hline \multicolumn{5}{|c|}{$\begin{array}{l}* \text { Mean } \pm \text { SD for continuous variables: } p \text { value was calculated by weighted linear regression model. } \\
\% \text { for categorical variables: } p \text { value was calculated by weighted chi-square test. }\end{array}$} \\
\hline \multicolumn{5}{|l|}{ NHANES sample. } \\
\hline \multicolumn{5}{|c|}{$\begin{array}{l}{ }^{\S} \text { BMI was calculated as the body weight in kilograms divided by the square of the height in meters. } \\
\text { BMI, body mass index; SBP, systolic blood pressure; DBP, diastolic blood pressure; TMFA, total } \\
\text { onounsaturated fatty acids; TPFA, total polyunsaturated fatty acids; TSFA, total saturated fatty acids; ALT, } \\
\text { anine aminotransferase; AST, aspartate aminotransferase; GGT, gamma glutamyl transferase; ACR, } \\
\text { bumin-creatinine ratio; eGFR, estimated glomerular filtration rate; SUA, serum uric acid; FBG, fasting blood } \\
\text { ucose; CKD, chronic kidney disease. }\end{array}$} \\
\hline
\end{tabular}

medicine to lower blood pressure (BP)." Participants who did not satisfy any of these criteria were defined as without hypertension. We defined diabetes using 1 of the following 5 criteria: previous diagnosis of diabetes, intake of antidiabetic medications or insulin, glycated hemoglobin level of $\geq 6.5 \%$, fasting blood glucose (FBG) level of $\geq 126 \mathrm{mg} / \mathrm{dL}(\geq 7.0 \mathrm{mmol} / \mathrm{L}$ ), or a 2-h glucose level of $\geq 200 \mathrm{mg} / \mathrm{dL}$ ( $\geq 11.1 \mathrm{mmol} / \mathrm{L}$ ) after an oral glucose tolerance test [17]. Participants who did not satisfy any of these 5 criteria were defined as without diabetes.

\section{Other Covariates}

Continuous variables included age (years), body mass index (BMI, $\mathrm{kg} / \mathrm{m}^{2}$ ), BP (mm $\mathrm{Hg}$ ), poverty to income ratio, dietary data (alcohol [g], caffeine [mg], calcium [mg], carbohydrate [g], cholesterol [mg], dietary fiber [g], energy [kcal], total monounsaturated fatty acids [g], total polyunsaturated fatty acids [g], total saturated fatty acids [g], total fat [g], protein [g]) and laboratory data (albumin $[\mathrm{g} / \mathrm{dL}]$, alanine aminotransferase $[\mathrm{U} / \mathrm{L}]$, aspartate aminotransferase $[\mathrm{U} / \mathrm{L}]$, gamma glutamyl transferase $[\mathrm{U} / \mathrm{L}]$, total cholesterol $[\mathrm{mg} / \mathrm{dL}]$, triglycerides $[\mathrm{mg} / \mathrm{dL}]$, homocysteine $[\mu \mathrm{mol} / \mathrm{L}]$, serum folate $[\mathrm{ng} / \mathrm{mL}]$ ). Categorical variables consisted of race/ethnicity (non-hispanic white, non-hispanic black, Mexican American, other hispanic or other), education (less than high school, high school/equivalent, or greater than high school), smoking (never smoker, former smoker, or current smoker), and physical activity (sedentary, low, moderate, high).

\section{Statistical Analysis and Missing Data}

The statistical analyses were performed according to the guidelines of the Centers for Disease Control and Prevention (https://wwwn.cdc.gov/nchs/nhanes/tutorials/default. aspx). We accounted for marked variance and used the proposed weighting methodology. Data are presented as mean \pm SD or proportions. We used weighted chi-square test (categorical variables) or weighted linear regression analyses (continuous variables) to calculate for differences among different SUA groups (tertiles). To investigate the association between SUA and CKD, our statistical analyses consisted of 3 main steps. Step 1: Univariate and multivariate binary logistic regression analyses were employed. We constructed 3 models: model 1 , no covariates were adjusted; model 2 , adjusted for sociodemographic data; model 3 , model $2+$ other covariates presented in Table 1. Step 2: To address for nonlinearity of SUA and CKD, 


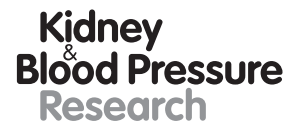

Research \begin{tabular}{l|l}
\hline Kidney Blood Press Res 2019;44:1036-1049 \\
\hline DOI: 10.1159/000502183 & $\begin{array}{l}\text { @ 2019 The Author(s). Published by S. Karger AG, Basel } \\
\text { www.karger.com/kbr }\end{array}$ \\
\hline
\end{tabular}

Hu et al.: Threshold Effects of SUA on CKD in Healthy Women

a generalized additive model and smooth curve fitting (penalized spline method) were conducted. If nonlinearity was detected, we first calculated the inflection point using recursive algorithm and then constructed a 2-piecewise binary logistic regression model on both sides of the inflection point. The threshold level was determined by choosing the inflection point which provided the maximum model likelihood, using the trial and error method, along with a log likelihood ratio test comparing the 1-line logistic regression model with a 2-piecewise logistic model to examine the statistical significance [18]. Step 3: The subgroup analyses were performed using stratified logistic regression analyses.

Of the 6,776 individuals in the analytic sample, we listed the missing data for each variable in online supplementary Table S1 (see www.karger.com/doi/10.1159/000502183 for all online suppl. material). For missing of covariates, we used multiple imputation, based on 5 replications and the Markov-chain Monte Carlo method in the SAS MI procedure, to maximize statistical power, and minimize bias that might occur covariates with missing data were excluded from data analyses $[19,20]$. In addition, we used sensitivity analysis to identify whether created complete data had significant difference from preimputation data (online suppl. Table S2). Our findings demonstrated that created complete data showed no significant difference from raw data.

To ensure the robustness of data analysis, we did the following sensitivity analysis: (1) we converted the SUA into a categorical variable and calculated the $p$ for trend. The purpose was to verify the results of SUA as the continuous variable and to observe the possibility of nonlinearity. (2) The main results were also performed by the imputed datasets and pooled by rubin's rule.

All the analyses were performed using the statistical package R (http://www.R-project. org, The R Foundation) and Empower (R; www.empowerstats.com; X\&Y Solutions, Inc., Boston, MA, USA). A $p$ value $<0.05$ was considered statistically significant.

\section{Results}

\section{Baseline Characteristics of Study Participants}

Based on the inclusion and exclusion criteria, a total of 6,776 women without hypertension and diabetes (mean age $38.3 \pm 17.9$ years; median age 34 [18-85] years) were selected for final data analysis. The weighted distribution of selective participants' sociodemographic characteristics and other covariates according to SUA tertiles is shown in Table 1 . The ranges of SUA for tertiles $1-3$ were $<4.5,4.5-5.7$, and $\geq 5.7 \mathrm{mg} / \mathrm{dL}$, respectively. There were significant differences between SUA tertiles, except for cholesterol intake, total polyunsaturated fatty acid intake, protein intake, and serum folate. Participants with the highest group of SUA T3 had higher values in age, BMI, SBP, DBP, alcohol, alanine aminotransferase, aspartate aminotransferase, gamma glutamyl transferase, ACR, FBG, 2-h glucose, glycated hemoglobin, total cholesterol, triglycerides, homocysteine and consisted of more non-hispanic black, high school, former and current smoker, sedentary, and low physical activity than those of the other groups.

\section{Association of CKD with SUA}

In this study, we constructed four models for analyzing the independent role of SUA in CKD. The effect values (ORs) and 95\% CIs for these three equations are listed in Table 2 . These effect sizes of models 2 and 3 were also pooled by Rubin's rule using imputation data (online suppl. Table S3). As shown in Table 2, the prevalence of CKD was 8.3\%. SUA was positively associated with CKD in different models. In fully-adjusted model, each $1 \mathrm{mg} / \mathrm{dL}$ of SUA higher was associated with $39 \%$ increased prevalence of CKD $(1.39,95 \%$ CI 1.23-1.58). We also 
Table 2. Relationship between SUA and CKD in different models

\begin{tabular}{|c|c|c|c|c|}
\hline & \multirow[t]{2}{*}{ Events, \% } & \multicolumn{3}{|l|}{ CKD, OR (95\% CI), $p$ value } \\
\hline & & model 1 & model 2 & model 3 \\
\hline Continuous & $561(8.3)$ & $1.64(1.53-1.76),<0.001$ & $1.46(1.32-1.61),<0.001$ & $1.39(1.23-1.58),<0.001$ \\
\hline \multicolumn{5}{|c|}{ Tertiles $(\mathrm{mg} / \mathrm{dL})$} \\
\hline $\mathrm{T} 1(<4.5)$ & $209(5.4)$ & Reference & Reference & Reference \\
\hline $\mathrm{T} 2(4.5-5.7)$ & $189(8.9)$ & $1.71(1.39-2.10),<0.001$ & 1.55 (1.21-1.99), 0.001 & 1.37 (1.02-1.84), 0.037 \\
\hline T3 ( $\geq 5.7)$ & $163(20.7)$ & $4.58(3.67-5.71),<0.001$ & $3.47(2.58-4.68),<0.001$ & $3.11(2.14-4.51),<0.001$ \\
\hline$p$ for trend & & $<0.001$ & $<0.001$ & $<0.001$ \\
\hline
\end{tabular}

Model 1: no covariates were adjusted.

Model 2 was adjusted for age, BMI, ethnicity, education status, smoking, alcohol consumption, physical activity, and poverty to income ratio.

Model 3 was adjusted for all covariables in model 2 plus adjusted for SBP, DBP, caffeine intake, calcium intake, carbohydrate intake, cholesterol intake, dietary fiber intake, energy intake, TSFAs intake, TPFAs intake, TSFAs intake, total fat intake, protein intake, albumin, ALT, AST, GGT, FBG, total cholesterol, triglycerides, homocysteine and serum folate.

BMI, body mass index; SBP, systolic blood pressure; DBP, diastolic blood pressure; TMFA, total monounsaturated fatty acids; TPFA, total polyunsaturated fatty acids; TSFA, total saturated fatty acids; ALT, alanine aminotransferase; AST, aspartate aminotransferase; GGT, gamma glutamyl transferase; SUA, serum uric acid; FBG, fasting blood glucose; CKD, chronic kidney disease.

converted SUA from a continuous variable to a categorical variable (tertile). Compared to participants with SUA of T1, there was a significant increased prevalence of CKD for the participants both with SUA of T2 and T3 (all $p<0.05$ ). $p$ for trend in the all models was significant. Additionally, the effect of ORs in different SUA groups was nonequal, suggesting that the association between SUA and CKD was likely to be nonlinear. The similar results could be observed in online supplementary Table S3.

\section{Nonlinear Relationship between SUA and CKD}

We also tried to use generalized additive model and penalized spline method to find the nonlinear relationship between SUA and CKD (Fig. 2). Adjusted smooth curve showed that the relationship between SUA and CKD was nonlinear. On the left of inflection point, the estimated dose-response curve was consistent with a horizontal line. The prevalence of increased CKD with SUA levels on the right of inflection point. Sensitivity analysis showed that the nonlinear trends of the data among pre- and post-imputation were approximately the same (Fig. 3). We fit the association between SUA and CKD using the standard binary logistic regression model and the 2-piecewise binary logistic regression model, respectively (Table 3 , online suppl. Table S4). The $p$ for log likelihood ratio test is $<0.001$, indicating that the twopiecewise binary logistic regression was more suitable for fitting the association between SUA and CKD. The inflection point of SUA was $4.5 \mathrm{mg} / \mathrm{dL}$. The ORs (95\% CIs) were 0.84 (0.661.08) on the left side of inflection point and 1.87 (1.56-2.24) on the right of inflection point, respectively. The results suggested that the prevalence of increased CKD with SUA levels only on the right of inflection point (SUA $>4.5 \mathrm{mg} / \mathrm{dL}$ ).

\section{Subgroup Analysis}

We further used all covariates presented in Table 1 as the stratification variables to observe the trend of effect sizes in these variables (online suppl. Table S5). Regardless of subgroup, SUA was positively associated with CKD, and the effect was more significant in the 


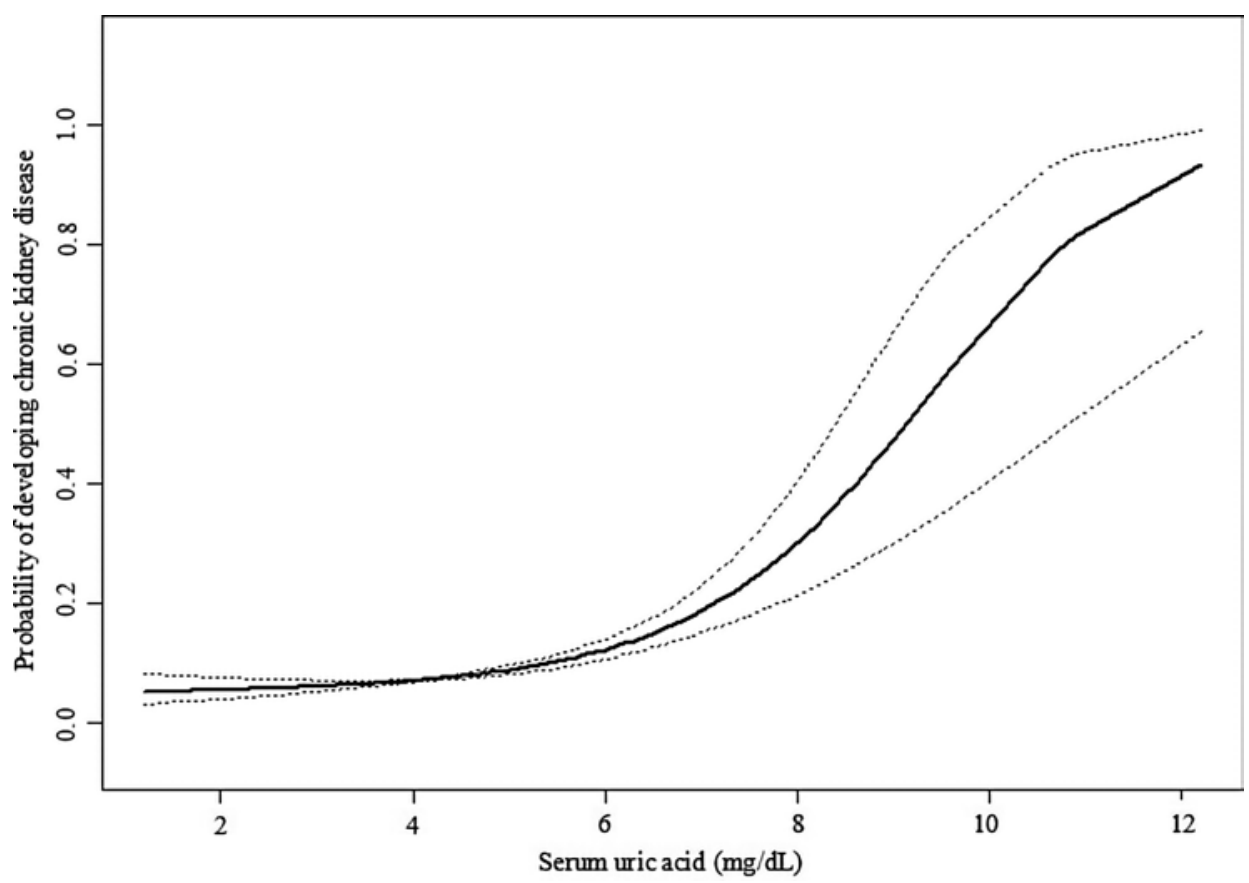

Fig. 2. Dose - response relationships between the probability of CKD and SUA. A threshold, nonlinear association between SUA and CKD was found $(p<0.001)$ in a generalized additive model. The solid line and dashed line represent the estimated values and their corresponding 95\% CIs. Adjustment factors included age, BMI, ethnicity, education status, smoking, alcohol consumption, physical activity, poverty to income ratio, SBP, DBP, caffeine intake, calcium intake, carbohydrate intake, cholesterol intake, dietary fiber intake, energy intake, total monounsaturated fatty acids intake, total polyunsaturated fatty acids intake, total saturated fatty acids intake, total fat intake, protein intake, albumin, alanine aminotransferase, aspartate aminotransferase, gamma glutamyl transferase, FBG, total cholesterol, triglycerides, homocysteine, and serum folate. SUA, serum uric acid; CKD, chronic kidney disease.

following subgroups: aged $>60$ years group $(18-40$ years: OR $1.11,95 \%$ CI $0.88-1.40 ; 40-60$ years: OR 1.22, 95\% CI 0.92-1.63; > 60 years: OR 2.03, 95\% CI 1.43-2.86, $p$ for interaction $=$ 0.002), never and former smoking group (never: OR 1.52, 95\% CI 1.25-2.86; former: OR 1.56, 95\% CI 1.07-2.28; current: OR 1.08, 95\% CI 0.80-1.47, $p$ for interaction $=0.009$ ), and FPG > $92 \mathrm{mg} / \mathrm{dL}$ group (<85 mg/dL: OR 1.16, 95\% CI 0.94-1.43; 85-92 mg/dL: OR 1.38, 95\% CI 1.10-1.72; >92 mg/dL: OR 1.84, 95\% CI 1.37-2.48, $p$ for interaction $=0.038$ ). However, the effect of SUA on CKD was consistent within race, education, physical activity, poverty to income ratio, $\mathrm{BMI}, \mathrm{SBP}, \mathrm{DBP}$, total cholesterol, triglycerides, and homocysteine groups (all $p$ for interaction $>0.05$ ).

\section{Discussion}

In the large selected sample of US women without hypertension and diabetes, we found SUA was positively and independently associated with CKD after adjusting other covariates. Each $1 \mathrm{mg} / \mathrm{dL}$ increase in SUA was associated with 39\% increased prevalence of CKD. Besides, we also found the nonlinearity between SUA and prevalence of CKD and discussed in depth. 


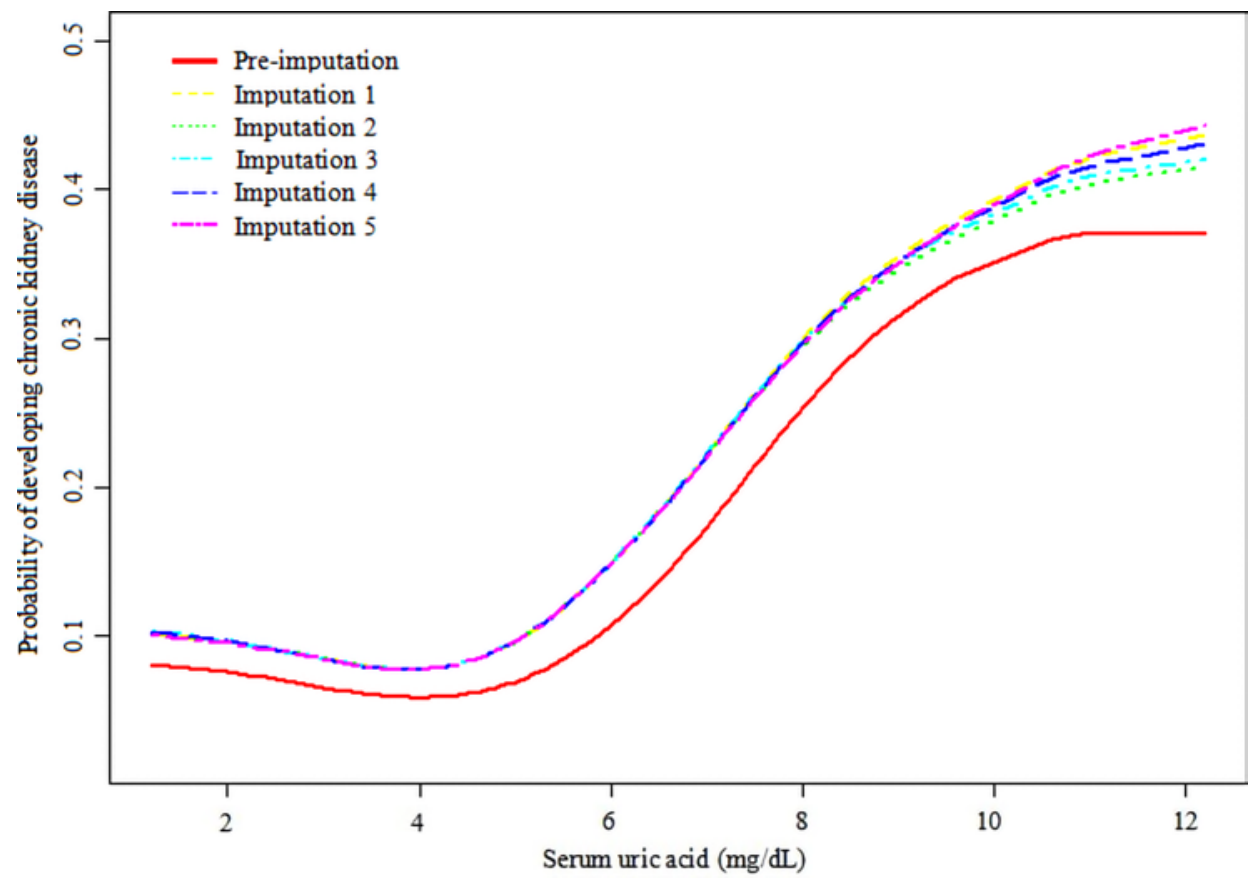

Fig. 3. Association between SUA and CKD among pre- and post-imputation data. A threshold, nonlinear association between SUA and CKD was found $(p<0.001)$ in a generalized additive model. Adjustment for age, BMI, ethnicity, education status, smoking, alcohol consumption, physical activity, poverty to income ratio, SBP, DBP, caffeine intake, calcium intake, carbohydrate intake, cholesterol intake, dietary fiber intake, energy intake, total monounsaturated fatty acids intake, total polyunsaturated fatty acids intake, total saturated fatty acids intake, total fat intake, protein intake, albumin, alanine aminotransferase, aspartate aminotransferase, gamma glutamyl transferase, FBG, total cholesterol, triglycerides, homocysteine, and serum folate. SUA, serum uric acid; CKD, chronic kidney disease.

The trends of the association between SUA and the prevalence of CKD were inconsistent on the left and right sides of the inflection point $(4.5 \mathrm{mg} / \mathrm{dL})$. The ORs $(95 \% \mathrm{CIs})$ were 0.84 $(0.66-1.08)$ on the left side of inflection point and 1.87 (1.56-2.24) on the right of inflection point, respectively, suggesting the threshold effects of SUA on the prevalence of CKD. Moreover, subgroup analyses showed that stronger associations between SUA and CKD were detected in elder women with never/former smoking and higher FBG levels.

Large longitudinal studies confirmed that hyperuricemia predicts the development of CKD. De Cosmo et al. [21] suggested that mild hyperuricemia was strongly associated with the risk of CKD in patients with type 2 diabetes. Chang et al. [22] and Mun et al. [23] also reported the consistent results. Hyperuricemia is associated with development and progression of CKD in adults. When kidney disease is present, decreased glomerular filtration may cause a rise in SUA and provides additional risk for progressive decline in kidney function. There are some explanations as to why high SUA levels affect the progression of kidney failure. Increased SUA levels can increase cyclooxygenase- 2 expression and lead to vascular smooth cell proliferation, causing hypertension and impairing kidney function [24, 25]. Furthermore, high SUA levels can activate the NLRP3 inflammasome and induce the production of interleukin-1 $\beta$, eventually stimulate the renal inflammation and fibrosis [26]. In contrast to our findings, several recent studies fail to identify an association between SUA levels and CKD [11, 12, 27]. 
Kidney

Blood Pressure

Research
Kidney Blood Press Res 2019;44:1036-1049

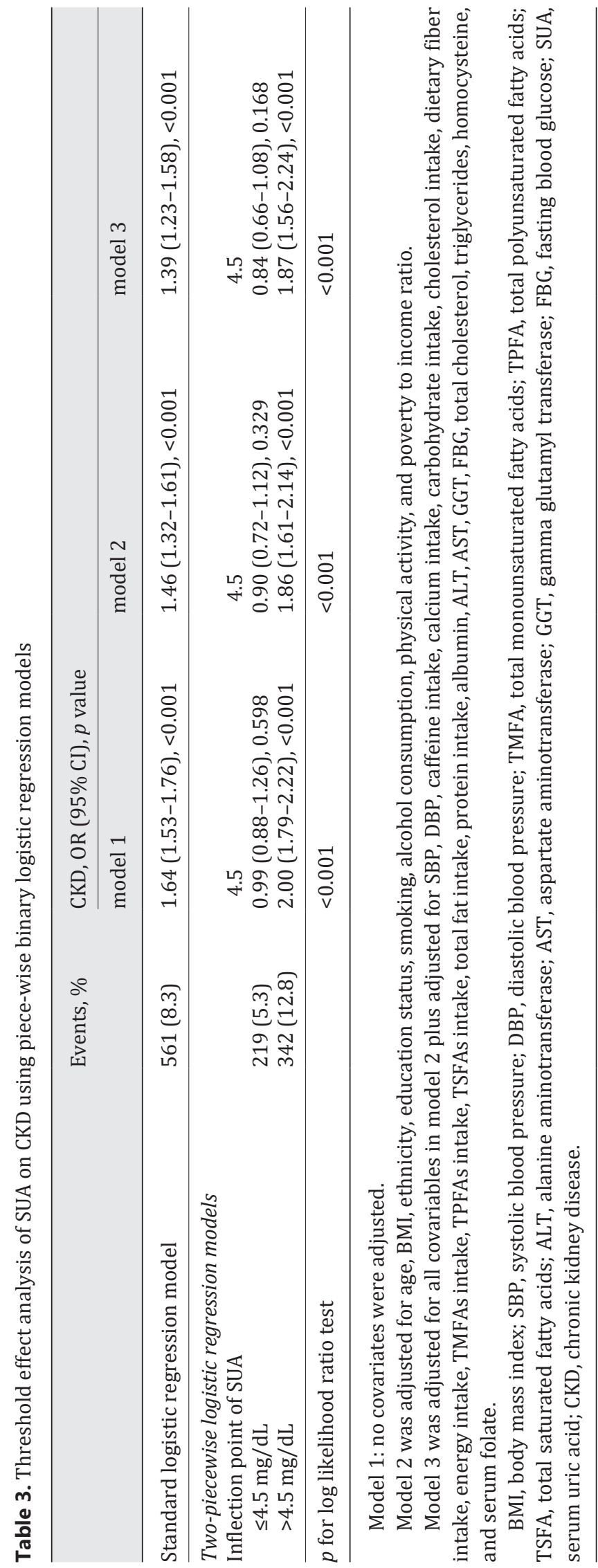




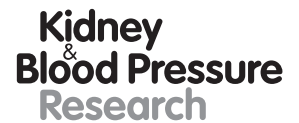

Kidney
Blood Pressure
Research \begin{tabular}{l|l}
\hline Kidney Blood Press Res 2019;44:1036-1049 \\
\hline DOI: 10.1159/000502183 & $\begin{array}{l}\text { @ 2019 The Author(s). Published by S. Karger AG, Basel } \\
\text { www.karger.com/kbr }\end{array}$ \\
\hline
\end{tabular}

Hu et al.: Threshold Effects of SUA on CKD in Healthy Women

These conflicting results may be attributed to the differences in cohort characteristics, sample size, and adjustment of confounders. Additionally, the reference values of SUA levels among these studies are still uneven and most studies categorize participants by quantiles, which markedly hamper the comparability and clinical applicability of these studies.

A few reports have shown that low SUA levels conferred an increased risk of CKD development. A study by Kanda et al. [28] showed that SUA had a U-shaped association with loss of kidney function in healthy Japanese. SUA levels of $<5.0$ and $<3.6 \mathrm{mg} / \mathrm{dL}$ in Japanese men and women, respectively, were reportedly considered as potential predictors of decreased kidney function. In addition, Wang et al. [29] reported that SUA levels $<2.0 \mathrm{mg} / \mathrm{dL}$ increased CKD incidence in Taiwan. However, in our study, we discussed the nonlinear relationship between SUA and CKD and found only SUA levels $>4.5 \mathrm{mg} / \mathrm{dL}$ were positively associated with CKD among US women without hypertension and diabetes, suggesting a threshold effects. Further research is needed to clarify the issue.

According to STROBE statement [30], subgroup analysis can make better use of data to reveal underlying truths. Our findings showed that SUA was positively associated with CKD in subgroups and some factors modified the associations. The results suggested that elder women (age $>60$ years) showed the stronger association between SUA and CKD. Age was a strong risk factor for CKD, with a study even reporting that the prevalence of CKD to increase dramatically with age [31]. Moreover, female hormones are known to affect SUA, which will be elevated in postmenopausal women [32]. Therefore, the effect of SUA levels on CKD was stronger in old age. In addition, we found that smoking status could modify the association of CKD with SUA. Epidemiologic studies have shown that smoking is associated with CKD [33, 34]. Smoking could promote endothelial dysfunction, oxidative stress, and inflammation with reduced kidney function $[35,36]$. However, in our study, we found that stronger associations between SUA and CKD were detected in women with never/former smoking, not current smoking. Perhaps because these participants who had some comorbidities gave up smoking. Current smokers often suggest that they are healthy. Further research is needed to clarify the issue. Interestingly, we also found that the effect of SUA on CKD was more significant in the high FBG group in the normal range. There was an increasing SUA with increasing FBG levels. The findings might be due to SUA strongly related to features of the metabolic syndrome, which might imply the presence of inflammation and insulin resistance, which are typical risk factors of CKD development, as well as outcomes of high SUA levels. Our results also suggested that prediabetic metabolism played an important role in CKD. A meta-analysis included 9 cohort studies with a total of 185,452 participants who were followed for a total of 835,146 person-years [37]. The result showed that prediabetes was modestly associated with an increase in CKD risk (relative ratio $1.12,95 \% \mathrm{CI}$ 1.02-1.21). Markus et al. [38] showed that prediabetes was associated with increased prevalence of CKD (OR 1.58, 95\% CI 1.10-2.25) among population without known type 2 diabetes.

Our study has some strengths. (1) This study was the first report to explore the association between SUA and CKD among US women without hypertension and diabetes; (2) in the study, we addressed the nonlinearity between SUA and CKD and further explained this nonlinearity; (3) this study is an observational study and therefore susceptible to potential confounding. We used strict statistical adjustment to minimize residual confounders; (4) the effect modifier factor analysis makes the use of data better and yield stable conclusion in different subgroups in this study; (5) we conducted sensitivity analyses to enhance the robustness of results.

There are some limitations in our study, which should be considered in the interpretation of results. First, due to the nature of cross-sectional study, it was difficult to estimate the causal association between SUA and CKD. Second, the study participants were US women without hypertension and diabetes; thus, the generalizability of the results to other populations remained to be verified. Third, as an observational study, we are unable to entirely rule out uncontrolled confounding or reverse causality. 


\section{Conclusion}

In summary, our study suggested threshold effects of SUA on the prevalence of CKD among US women without hypertension and diabetes. SUA levels $>4.5 \mathrm{mg} / \mathrm{dL}$ may be positively associated with CKD. The results suggest that we need to strengthen the management of SUA in healthy women to reduce the risk of CKD. Further researches are needed to elucidate actual role of SUA in CKD and underlying potential mechanisms.

\section{Acknowledgments}

A special thanks to all of the NHANES participants who gave freely of their time to make this and other studies possible. Moreover, we gratefully thank Empower U team of the Department of Epidemiology and Biostatistics, X\&Y solutions Inc., in Boston for their contribution to the statistical support.

\section{Statement of Ethics}

All procedures performed in studies involving human participants were in accordance with the ethical standards of the institutional and/or national research committee and with the 1964 Helsinki Declaration and its later amendments or comparable ethical standards. Informed consent was obtained from all individual participants included in the study.

\section{Disclosure Statement}

G.H., Y.B., T.C., S.T., and L.H.: The authors declare that they have no conflict of interest.

\section{Funding Sources}

The authors would like to acknowledge the support from the National Natural Science Foundation of China $(81573118,91743114,81872598)$ and the innovation fund of the excellent doctoral graduate students of Peking University Health Science Center.

\section{Author Contribution}

G.H. and L.H.: conceived the study concept. G.H., Y.B., and L.H.: analyzed the data. G.H.: drafted the report. T.C., S.T. and L.H.: provided critical revisions for important intellectual content. All authors took part in the interpretation of the data. All authors have read and approved the final manuscript.

\section{Novelty Statements}

We found that SUA levels $>4.5 \mathrm{mg} / \mathrm{dL}$ were positively and independently associated with CKD among US women without hypertension and diabetes. The results suggest that we need to strengthen the management of SUA in healthy women to reduce the risk of CKD. 
Hu et al.: Threshold Effects of SUA on CKD in Healthy Women

\section{References}

1 Levey AS, Atkins R, Coresh J, Cohen EP, Collins AJ, Eckardt KU, et al. Chronic kidney disease as a global public health problem: approaches and initiatives - a position statement from Kidney Disease Improving Global Outcomes. Kidney Int. 2007 Aug; 72(3):247-59.

2 Sud M, Tangri N, Pintilie M, Levey AS, Naimark D. Risk of end-stage renal disease and death after cardiovascular events in chronic kidney disease. Circulation. 2014 Aug;130(6):458-65.

3 Gansevoort RT, Correa-Rotter R, Hemmelgarn BR, Jafar TH, Heerspink HJ, Mann JF, et al. Chronic kidney disease and cardiovascular risk: epidemiology, mechanisms, and prevention. Lancet. 2013 Jul;382(9889): 339-52.

4 Muntner P, Anderson A, Charleston J, Chen Z, Ford V, Makos G, et al.; Chronic Renal Insufficiency Cohort (CRIC) Study Investigators. Hypertension awareness, treatment, and control in adults with CKD: results from the Chronic Renal Insufficiency Cohort (CRIC) Study. Am J Kidney Dis. 2010 Mar;55(3):441-51.

5 El Minshawy 0, Ghabrah T, El Bassuoni E. Diabetic nephropathy as a cause of end-stage renal disease in Tabuk area, Saudi Arabia: a four-year study. Saudi J Kidney Dis Transpl. 2014 Sep;25(5):1105-9.

6 Feig DI, Kang DH, Johnson RJ. Uric acid and cardiovascular risk. N Engl J Med. 2008 Oct;359(17):1811-21.

7 Fang J, Alderman MH. Serum uric acid and cardiovascular mortality the NHANES I epidemiologic follow-up study, 1971-1992. National Health and Nutrition Examination Survey. JAMA. 2000 May;283(18):2404-10.

8 Chen X, Li Y, Sheng CS, Huang QF, Zheng Y, Wang JG. Association of serum uric acid with aortic stiffness and pressure in a Chinese workplace setting. Am J Hypertens. 2010 Apr;23(4):387-92.

9 Iseki K, Ikemiya Y, Inoue T, Iseki C, Kinjo K, Takishita S. Significance of hyperuricemia as a risk factor for developing ESRD in a screened cohort. Am J Kidney Dis. 2004 Oct;44(4):642-50.

10 Rodenbach KE, Schneider MF, Furth SL, Moxey-Mims MM, Mitsnefes MM, Weaver DJ, et al. Hyperuricemia and Progression of CKD in Children and Adolescents: The Chronic Kidney Disease in Children (CKiD) Cohort Study. Am J Kidney Dis. 2015 Dec;66(6):984-92.

11 Madero M, Sarnak MJ, Wang X, Greene T, Beck GJ, Kusek JW, et al. Uric acid and long-term outcomes in CKD. Am J Kidney Dis. 2009 May;53(5):796-803.

12 Goicoechea M, Garcia de Vinuesa S, Verdalles U, Verde E, Macias N, Santos A, et al. Allopurinol and progression of CKD and cardiovascular events: long-term follow-up of a randomized clinical trial. Am J Kidney Dis. 2015 Apr;65(4):543-9.

13 Selvin E, Manzi J, Stevens LA, Van Lente F, Lacher DA, Levey AS, et al. Calibration of serum creatinine in the National Health and Nutrition Examination Surveys (NHANES) 1988-1994, 1999-2004. Am J Kidney Dis. 2007 Dec;50(6):918-26.

14 Levey AS, Stevens LA, Schmid CH, Zhang YL, Castro AF 3rd, Feldman HI, et al.; CKD-EPI (Chronic Kidney Disease Epidemiology Collaboration). A new equation to estimate glomerular filtration rate. Ann Intern Med. 2009 May;150(9):604-12.

15 Chavers BM, Simonson J, Michael AF. A solid phase fluorescent immunoassay for the measurement of human urinary albumin. Kidney Int. 1984 Mar;25(3):576-8.

16 Levey AS, de Jong PE, Coresh J, El Nahas M, Astor BC, Matsushita K, et al. The definition, classification, and prognosis of chronic kidney disease: a KDIGO Controversies Conference report. Kidney Int. 2011 Jul;80(1): 17-28.

17 American Diabetes Association. Standards of medical care in diabetes-2014. Diabetes Care. 2014 Jan;37 Suppl 1:S14-80.

18 Yu X, Cao L, Yu X. Elevated cord serum manganese level is associated with a neonatal high ponderal index. Environ Res. 2013 Feb;121:79-83.

19 Bernhardt PW. Model validation and influence diagnostics for regression models with missing covariates. Stat Med. 2018 Apr;37(8):1325-42.

20 Park SY, Freedman ND, Haiman CA, Le Marchand L, Wilkens LR, Setiawan VW. Association of Coffee Consumption With Total and Cause-Specific Mortality Among Nonwhite Populations. Ann Intern Med. 2017 Aug;167(4):228-35.

21 De Cosmo S, Viazzi F, Pacilli A, Giorda C, Ceriello A, Gentile S, et al.; AMD-Annals Study Group. Serum Uric Acid and Risk of CKD in Type 2 Diabetes. Clin J Am Soc Nephrol. 2015 Nov;10(11):1921-9.

22 Chang YH, Lei CC, Lin KC, Chang DM, Hsieh CH, Lee YJ. Serum uric acid level as an indicator for CKD regression and progression in patients with type 2 diabetes mellitus-a 4.6-year cohort study. Diabetes Metab Res Rev. 2016 Sep;32(6):557-64.

23 Mun KH, Yu GI, Choi BY, Kim MK, Shin MH, Shin DH. Effect of Uric Acid on the Development of Chronic Kidney Disease: The Korean Multi-Rural Communities Cohort Study. J Prev Med Public Health. 2018 Sep;51(5):24856.

24 Johnson RJ, Segal MS, Srinivas T, Ejaz A, Mu W, Roncal C, et al. Essential hypertension, progressive renal disease, and uric acid: a pathogenetic link? J Am Soc Nephrol. 2005 Jul;16(7):1909-19.

25 Sánchez-Lozada LG, Soto V, Tapia E, Avila-Casado C, Sautin YY, Nakagawa T, et al. Role of oxidative stress in the renal abnormalities induced by experimental hyperuricemia. Am J Physiol Renal Physiol. 2008 Oct; 295(4):F1134-41.

26 Kim IY, Lee DW, Lee SB, Kwak IS. The role of uric acid in kidney fibrosis: experimental evidences for the causal relationship. BioMed Res Int. 2014;2014:638732. 
27 Nacak H, van Diepen M, de Goeij MC, Rotmans JI, Dekker FW; PREPARE-2 study group. Uric acid: association with rate of renal function decline and time until start of dialysis in incident pre-dialysis patients. BMC Nephrol. 2014 Jun;15(1):91.

28 Kanda E, Muneyuki T, Kanno Y, Suwa K, Nakajima K. Uric acid level has a U-shaped association with loss of kidney function in healthy people: a prospective cohort study. PLoS One. 2015 Feb;10(2):e0118031.

29 Wang S, Shu Z, Tao Q, Yu C, Zhan S, Li L. Uric acid and incident chronic kidney disease in a large health check-up population in Taiwan. Nephrology (Carlton). 2011 Nov; 16(8):767-76.

30 Vandenbroucke JP, von Elm E, Altman DG, Gøtzsche PC, Mulrow CD, Pocock SJ, et al.; STROBE Initiative. Strengthening the Reporting of Observational Studies in Epidemiology (STROBE): explanation and elaboration. PLoS Med. 2007 Oct;4(10):e297.

31 Park JI, Baek H, Jung HH. Prevalence of Chronic Kidney Disease in Korea: the Korean National Health and Nutritional Examination Survey 2011-2013. J Korean Med Sci. 2016 Jun;31(6):915-23.

32 Takiue Y, Hosoyamada M, Kimura M, Saito H. The effect of female hormones upon urate transport systems in the mouse kidney. Nucleosides Nucleotides Nucleic Acids. 2011 Feb;30(2):113-9.

33 Shankar A, Klein R, Klein BE. The association among smoking, heavy drinking, and chronic kidney disease. Am J Epidemiol. 2006 Aug;164(3):263-71.

34 Fox CS, Larson MG, Leip EP, Culleton B, Wilson PW, Levy D. Predictors of new-onset kidney disease in a community-based population. JAMA. 2004 Feb;291(7):844-50.

35 Jaimes EA, Tian RX, Raij L. Nicotine: the link between cigarette smoking and the progression of renal injury? Am J Physiol Heart Circ Physiol. 2007 Jan;292(1):H76-82.

36 Jaimes EA, DeMaster EG, Tian RX, Raij L. Stable compounds of cigarette smoke induce endothelial superoxide anion production via NADPH oxidase activation. Arterioscler Thromb Vasc Biol. 2004 Jun;24(6):1031-6.

37 Echouffo-Tcheugui JB, Narayan KM, Weisman D, Golden SH, Jaar BG. Association between prediabetes and risk of chronic kidney disease: a systematic review and meta-analysis. Diabet Med. 2016 Dec;33(12):1615-24.

38 Markus MR, Ittermann T, Baumeister SE, Huth C, Thorand B, Herder C, et al. Prediabetes is associated with microalbuminuria, reduced kidney function and chronic kidney disease in the general population: The KORA (Cooperative Health Research in the Augsburg Region) F4-Study. Nutr Metab Cardiovasc Dis. 2018 Mar;28(3): 234-42. 